\title{
The Role of Flt4 in Skin Protection against UVB Radiation: A System Biology Approach
}

\author{
Babak Arjmand $^{1 \oplus}$, Mostafa Rezaei Tavirani ${ }^{2 *}{ }^{\circledR}$, Mohammadreza Razzaghi $^{3}{ }^{\circledR}$, Mohammad Rostami-Nejad $^{4}$, \\ Mostafa Hamdieh ${ }^{5}$, Abdolrahim Nikzamir ${ }^{6}$
}

${ }^{1}$ Cell Therapy and Regenerative Medicine Research Center, Endocrinology and Metabolism Molecular-Cellular Sciences Institute, Tehran University of Medical Sciences, Tehran, Iran

${ }^{2}$ Proteomics Research Center, Faculty of Paramedical Sciences, Shahid Beheshti University of Medical Sciences, Tehran, Iran

${ }^{3}$ Laser Application in Medical Sciences Research Center, Shahid Beheshti University of Medical Sciences, Tehran, Iran ${ }^{4}$ Gastroenterology and Liver Diseases Research Center, Research Institute for Gastroenterology and Liver Diseases, Shahid Beheshti University of Medical Sciences, Tehran, Iran

${ }^{5}$ Department of Psychosomatic, Taleghani Hospital, Faculty of Medicine, Shahid Beheshti University of Medical Sciences, Tehran, Iran

${ }^{6}$ Faculty of Medicine, Shahid Beheshti University of Medical Sciences, Tehran, Iran

\section{*Correspondence to}

Mostafa Rezaei Tavirani

Proteomics Research Center

Faculty of Paramedical Sciences,

Shahid Beheshti University of

Medical Sciences, Tehran, Iran.

Email: tavirany@yahoo.com

Published online December 30, 2020

\begin{abstract}
Background: Although the application of ultraviolet B (UVB) in phototherapy of human skin is a common therapeutic method, it is known as a risk factor for skin cancer. This study aims to assess the role of differentially expressed genes (DEGs) to find the critical one that is mainly responsible for skin protection against UVB radiation.

Methods: The gene expression profiles of irradiated mice by UVB that issued skin protection against exposure are extracted from Gene Expression Omnibus (GEO) and analyzed by GEO2R. The significant DEGs are assessed via gene ontology (GO) analysis and the critical individuals are investigated via action mapping.

Results: Thirty-eight significant DEGs that provide skin resistance against UVB irradiation were determined. Among the query DEGs, 26 individuals were related to 43 biological terms. Flt4, F3, Tspan6, Cblb, and Itgb6 were highlighted as the critical DEGs to promote skin protection against UVB irradiation.

Conclusion: The finding indicates that Flt4 is the key DEG that is mainly responsible for protecting skin from UVB exposure.

Keywords: UVB; Skin; Mouse; Gene ontology; Flt4.
\end{abstract}

\section{Introduction}

Human skin receives ultraviolet (UV) radiation from sunlight, the prominent factor in promoting skin cancer. Since UV rays cannot penetrate into the deep part of the body, skin is the main target for UV radiation. ${ }^{1}$ Three types of UV light have been introduced as UVA, UVB, and UVC that are discriminated based on their wavelength. UVC (180-280 nm) has the highest level of energy while UVA (320-400 nm) has the lowest amounts of energy. The middle energy level belongs to the UVB (280-320 nm). ${ }^{2}$ UVB is known as a risk factor in promoting human skin cancer. The application of UVB in phototherapy of skin disorders such as vitiligo, cutaneous T-cell lymphoma, atopic dermatitis, alopecia, and psoriasis is accompanied by this question that 'Is using UVB phototherapy harmful for the patients'?3-6

Previous studies have shown that each kind of human body damage is accompanied by large changes in the expression of different genes in hurt individuals. There are various accessible data banks that store the differential expressed genes related to diseases and disorders. Gene expression Omnibus is the source that provides access to the numerous gene expression profiles. ${ }^{7,8}$ The analysis of the large numbers of differentially expressed genes (DEGs) implies the application of bioinformatics tools to discriminate the query DEGs based on the impact and importance of gene products relative to each other. ${ }^{9,10}$ In such analysis among the large numbers of DEGs, the limited individuals are introduced as the critical genes which are responsible for the main part of changes. The gene ontology approach is a useful method for determining the biological processes, cellular components, molecular function, and biochemical pathways that are involved with the studied DEGs. ${ }^{11,12}$ Another useful tool is action

Please cite this article as follows: Arjmand B, Rezaei Tavirani M, Razzaghi M, Rostami-Nejad M, Hamdieh M, Nikzamir A. The role of flt4 in skin protection against uvb radiation: a system biology approach. J Lasers Med Sci. 2020;11(suppl 1):S30-S36. doi:10.34172/jlms.2020. S5. 
map analysis that determines types of relationships such as activation, inhibition, and regulation actions between the studied set of genes. ${ }^{13}$ In the present study, a set of genes that are involved in the protection of mouse skin against UVB irradiation are analyzed via the gene ontology method to find the crucial one.

\section{Materials and Methods}

GSE79073/GPL6246 was extracted from GEO (a public functional genomics data repository). As it is reported, two groups of mice (each group includes 3 mice) were exposed to a single dose of $80 \mathrm{~mJ} / \mathrm{cm}^{2}$ of UVB (emission range, 280-300; peak $324 \mathrm{~nm}$ ) radiation. The first group which was a wild type in this study is shown as group 1 and it is considered as the control group and the second group (group 2) included $\mathrm{CBL}-\mathrm{b} \%$ mice, considered as the sample group. Dorsal skin RNA samples of two studied groups before and 24 hours after exposure to UV were collected and analyzed by an Affymetrix Muse Gene 1.0 ST array. The details of methods are described in the report by Singh et al, published in a paper in $2018 .{ }^{14}$ In the present study, gene expression profiles of the two groups are analyzed via GEO2R software. First of all, the 6 gene expression profiles were statistically matched. Among all DEGs, 250 top individuals (based on $P$ value) were extracted by GEO2R software. Considering fold change (FC) more than 1.5 and $P$ value $<0.001$, the significant DEGs were identified among the selected 250 top DEGs. The significant uncharacterized DEGs were omitted. If, for a certain spot, several genes were suggested, the individuals with the first one were considered and the others were not included in the next step of the analysis.

Gene ontology analysis via the ClueGo application of Cytoscpe software ${ }^{15}$ was applied to identify pathways, cellular components, biological processes, and molecular function which were related to the 38 significant DEGs. The biological terms which included at least 6 genes and $P$ value $<0.05$ were considered for more analysis. The frequency of involved biological terms was identified for each significant DEG. CluPedia, the other plugin of Cytoscape,${ }^{16}$ was applied to determine the action map for the 38 significant DEGs.

\section{Results}

Statistical matching of gene expression profiles is shown in Figure 1. As it is depicted in the figure distributions of gene expression amounts of the samples are median center and can be matched. The 38 significant DEGs among the 250 extracted DEGs were included in the study. The significant DEGs and their properties are presented in Table 1 and Figure 2.

As it is depicted in Tables 2-4, gene ontology analysis revealed that 26 significant DEGs among all query DEGs are involved in 17 cellular components, 24 biological processes and pathways, and 2 molecular function terms. The numbers and titles of the involved DEGs and also

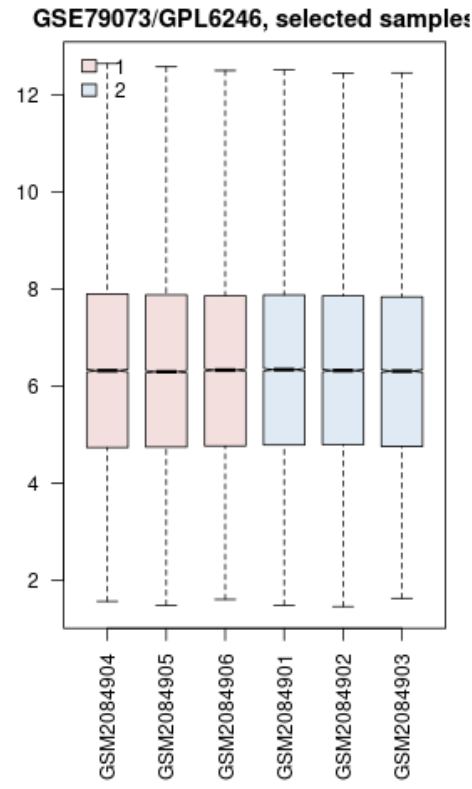

Figure 1. Six Gene Expression Profiles of the Two Irradiated Studied Groups of Mice Including the Wild Types (Group 1) (GSM208494-6) and CBL-b/ Mice (Group 2) (GSM2084901-3). The normalized values of the vertical axis refer to the frequency of gene expression values.

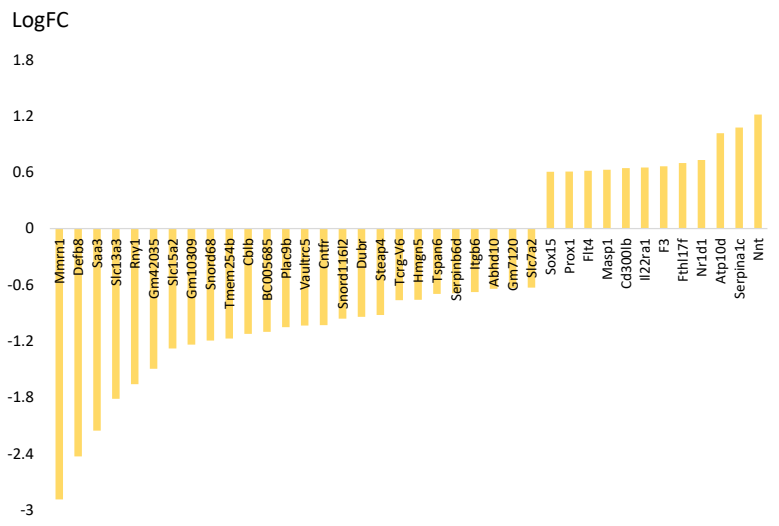

Figure 2. The Logarithm of Fold Change Is Illustrated for the 38 Significant DEGs. The minimum fold change is 1.5. Positive and negative values refer to up- and down-regulation in the CBL-b\% mice relative to the wild-type animals.

the percentage of participation of DEGs in the biological terms are presented in Tables 2-4.

For better understanding, the 26 DEGs that are involved in the biological terms and the frequency of the related biological terms for the individual DEGs are tabulated in Table 5.

The action map including activation, inhibition, regulation, binding, and reaction, for the 38 significant DEGs was provided by the CluePedia application of Cytoscape software. Action map presentation (see Figure 3) revealed that Flt4, F3, Tspan6, Cntfr, Nr1d1, Cblb, Itgb6, and Masp1 are connected with different types of actions. As it is presented in Figure 3, Flt4 not only activates Cblb but also inhibits this gene. Flt4 was up-regulated by Itgb6, 
Table 1. List of 38 DEGs That Differentiate the Two Studied Groups of Mice.

\begin{tabular}{|c|c|c|}
\hline Gene Symbol & Gene Title & $\log \mathrm{FC}$ \\
\hline Nnt & Nicotinamide nucleotide transhydrogenase & 1.214 \\
\hline Serpina 1c & $\begin{array}{l}\text { Serine (or cysteine) peptidase inhibitor, clade A, } \\
\text { member } 1 C\end{array}$ & 1.076 \\
\hline Atp10d & ATPase, class V, type $10 \mathrm{D}$ & 1.015 \\
\hline Nr1d1 & $\begin{array}{l}\text { Nuclear receptor subfamily } 1 \text {, group D, } \\
\text { member } 1\end{array}$ & 0.731 \\
\hline Fth $/ 17 f$ & Ferritin, heavy polypeptide-like 17 , member $\mathrm{F}$ & 0.697 \\
\hline F3 & Coagulation factor III & 0.663 \\
\hline II22ra1 & Interleukin 22 receptor, alpha 1 & 0.651 \\
\hline Cd300lb & CD300 molecule like family member B & 0.643 \\
\hline Masp1 & Mannan-binding lectin serine peptidase 1 & 0.626 \\
\hline Flt4 & FMS-like tyrosine kinase 4 & 0.616 \\
\hline Prox 1 & Prospero homeobox 1 & 0.608 \\
\hline Sox 15 & SRY (sex-determining region Y)-box 15 & 0.606 \\
\hline S/C7a2 & $\begin{array}{l}\text { Solute carrier family } 7 \text { (cationic amino acid } \\
\text { transporter, } y+\text { system), member } 2\end{array}$ & -0.631 \\
\hline Gm>120 & Predicted gene 7120 & -0.636 \\
\hline Abhd10 & Abhydrolase domain containing 10 & -0.643 \\
\hline Itgb6 & Integrin beta 6 & -0.677 \\
\hline Serpinb6d & $\begin{array}{l}\text { Serine (or cysteine) peptidase inhibitor, clade B, } \\
\text { member } 6 d\end{array}$ & -0.69 \\
\hline Tspan6 & Tetraspanin 6 & -0.697 \\
\hline Hmgn5 & $\begin{array}{l}\text { High-mobility group nucleosome binding } \\
\text { domain } 5\end{array}$ & -0.76 \\
\hline Tcrg-V6 & T-cell receptor gamma, variable 6 & -0.764 \\
\hline Steap4 & STEAP family member 4 & -0.922 \\
\hline Dubr & Dppa2 upstream binding RNA & -0.942 \\
\hline Snord116/2 & Small nucleolar RNA, C/D box 116-like 2 & -0.961 \\
\hline Cntfr & Ciliary neurotrophic factor receptor & -1.031 \\
\hline Vaultrc5 & Vault RNA component 5 & -1.034 \\
\hline Plac9b & Placenta specific $9 \mathrm{~b}$ & -1.051 \\
\hline BC005685 & cDNA sequence BC005685 & -1.101 \\
\hline Cblb & Casitas B-lineage lymphoma b & -1.123 \\
\hline Tmem $254 b$ & Transmembrane protein $254 \mathrm{~b}$ & -1.174 \\
\hline Snord68 & Small nucleolar RNA, C/D box 68 & -1.195 \\
\hline Gm10309 & predicted gene 10309 & -1.237 \\
\hline Slc15a2 & $\begin{array}{l}\text { Solute carrier family } 15(\mathrm{H}+\text { /peptide } \\
\text { transporter), member } 2\end{array}$ & -1.28 \\
\hline Gm42035 & Predicted gene, 42035 & -1.496 \\
\hline Rny 1 & RNA, Y1 small cytoplasmic, Ro-associated & -1.66 \\
\hline Slc13a3 & $\begin{array}{l}\text { Solute carrier family } 13 \text { (sodium-dependent } \\
\text { dicarboxylate transporter), member } 3\end{array}$ & -1.816 \\
\hline Saa3 & Serum amyloid A 3 & -2.155 \\
\hline Defb8 & Defensin beta 8 & -2.429 \\
\hline Mmrn 1 & Multimerin 1 & -2.888 \\
\hline
\end{tabular}

Note. $P$ value $<0.001$ and fold change (FC) more than 1.5 were considered. Positive and negative values refer to up- and down-regulation in the CBL-b/ mice relative to the wild types.
Cblb, and Prpx1 and up-regulates Itgb6 and Cblb.

\section{Discussion}

There are numerous documents about the mechanism of radiation (especially UV radiation) effect on $\operatorname{skin}^{17-}$ ${ }^{19}$ but more investigations should be planned to achieve clear presentation of the molecular mechanism which is related to the damages and also repair processes. In the present study, gene expression changes of dorsal skin of mice in the absence of CBL-b activity (CBL-b-/-mouse) after exposure to UVB in comparison with the wild types were investigated. Singh et al. have reported that in the absence of CBL-b activity, UVB irradiation leads to lower numbers of DNA photoproducts and sunburn cells. ${ }^{14}$ We assessed the genes that are possibly responsible for this protection.

As it is presented in the result part, there are 38 significant DEGs that discriminate the studied samples. GO analysis highlighted 26 DEGs among the 38 significant DEGs which were involved in the related biological terms. Action map analysis revealed that 8 DEGs among 26 individuals were connected to each other via action roles. A comparison between Table 5 and Figure 3 indicates that $63 \%$ of genes that are connected in the action map including Flt4, F3, Tspan6, Cblb, and Itgb6 are characterized with the "frequency of the involved biological terms" above $\% 50$ in Table 5. It seems that these 5 genes are the critical individuals that play a significant role in protection against UVB radiation. The illustrated connections in Figure 3 indicate that connections between Flt4, Tspan6, Cblb, and Itgb6 are so important and the genes are linked in a complex pattern of connections.

Tspan6 and Itgb6 up-regulate each other and this reciprocal regulation corresponds with the expression change of these dysregulated genes. As it is represented in Table 1 and Figure 2, Tspan6 and Itgb6 are down-
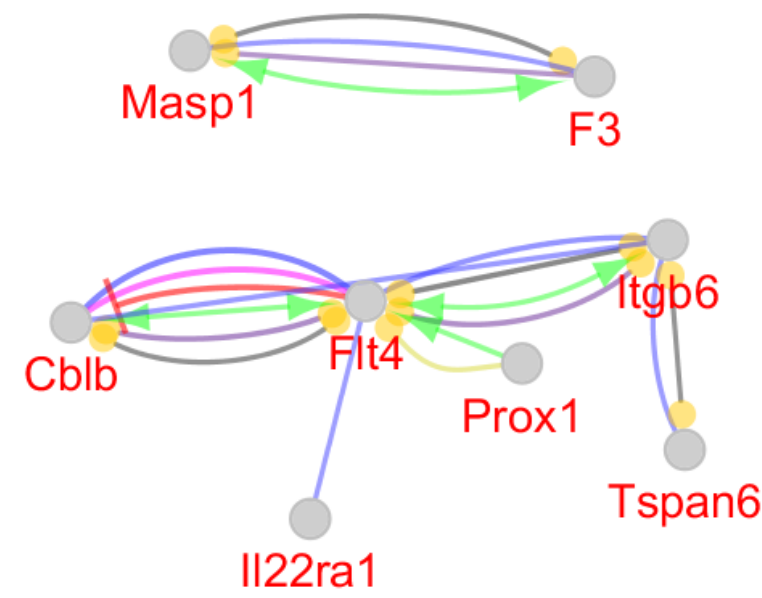

Figure 3. The Action Map of 38 Significant DEGs. Green, red, yellow, blue, purple, and black refer to activation, inhibition, regulation, binding, reaction, and catalysis actions respectively. 
Table 2. Seventeen Cellular Component Terms That Are Related to the Several Queried DEGs

\begin{tabular}{|c|c|c|c|}
\hline GO Term & $\%$ AGs & NGs & Associated Genes \\
\hline Extracellular region part & 0.30 & 11 & [Cntfr, F3, Itgb6, Masp1, Mmrn1, Saa3, Serpina1c, Slc13a3, Slc15a2, Steap4, Tspan6] \\
\hline Membrane part & 0.28 & 15 & $\begin{array}{l}\text { [Atp10d, Cblb, Cd300lb, Cntfr, F3, Flt4, Il22ra1, Itgb6, Nnt, Slc13a3, Slc15a2, Slc7a2, Steap4, } \\
\text { Tmem254b, Tspan6] }\end{array}$ \\
\hline Extracellular space & 0.29 & 10 & [Cntfr, F3, Itgb6, Masp1, Saa3, Serpina1c, Slc13a3, Slc15a2, Steap4, Tspan6] \\
\hline Intrinsic component of membrane & 0.32 & 15 & $\begin{array}{l}\text { [Atp10d, Cd300lb, Cntfr, F3, Flt4, Il22ra1, Itgb6, Nnt, Slc13a3, Slc15a2, Slc7a2, Steap4, } \\
\text { Tmem254b, Tspan6] }\end{array}$ \\
\hline Extracellular organelle & 0.27 & 7 & [F3, Itgb6, Serpina1c, Slc13a3, Slc15a2, Steap4, Tspan6] \\
\hline Cell periphery & 0.30 & 13 & $\begin{array}{l}\text { [Atp10d, Cblb, Cd300lb, Cntfr, F3, Flt4, Il22ra1, Itgb6, Slc13a3, Slc15a2, Slc7a2, Steap4, } \\
\text { Tspan6] }\end{array}$ \\
\hline Plasma membrane & 0.31 & 13 & $\begin{array}{l}\text { [Atp10d, Cblb, Cd300lb, Cntfr, F3, Flt4, Il22ra1, Itgb6, Slc13a3, Slc15a2, Slc7a2, Steap4, } \\
\text { Tspan6] }\end{array}$ \\
\hline Organelle part & 0.11 & 8 & [Atp10d, Cblb, Hmgn5, Masp1, Nnt, Nr1d1, Sox15, Steap4] \\
\hline Integral component of membrane & 0.33 & 15 & $\begin{array}{l}\text { [Atp10d, Cd300lb, Cntfr, F3, Flt4, Il22ra1, Itgb6, Nnt, Slc13a3, Slc15a2, Slc7a2, Steap4, } \\
\text { Tmem254b, Tspan6] }\end{array}$ \\
\hline Plasma membrane part & 0.31 & 7 & [Cntfr, Flt4, Itgb6, Slc13a3, Slc15a2, Steap4, Tspan6] \\
\hline Extracellular vesicle & 0.27 & 7 & [F3, Itgb6, Serpina1c, Slc13a3, Slc15a2, Steap4, Tspan6] \\
\hline Extracellular exosome & 0.28 & 7 & [F3, Itgb6, Serpina1c, Slc13a3, Slc15a2, Steap4, Tspan6] \\
\hline Intrinsic component of plasma membrane & 0.59 & 7 & [Cntfr, Flt4, Itgb6, Slc13a3, Slc15a2, Steap4, Tspan6] \\
\hline Integral component of plasma membrane & 0.63 & 7 & [Cntfr, Flt4, Itgb6, Slc13a3, Slc15a2, Steap4, Tspan6] \\
\hline Cell part & 0.16 & 22 & $\begin{array}{l}\text { [Abhd10, Atp10d, Cblb, Cd300lb, Cntfr, F3, Flt4, Hmgn5, Il22ra1, Itgb6, Masp1, Nnt, Nr1d1, } \\
\text { Prox1, Saa3, Serpina1c, Slc13a3, Slc15a2, Slc7a2, Sox15, Steap4, Tspan6] }\end{array}$ \\
\hline Membrane-bounded organelle & 0.17 & 18 & $\begin{array}{l}\text { [Abhd10, Atp10d, Cblb, F3, Flt4, Hmgn5, Itgb6, Masp1, Nnt, Nr1d1, Prox1, Serpina1c, } \\
\text { Slc13a3, Slc15a2, Slc7a2, Sox15, Steap4, Tspan6] }\end{array}$ \\
\hline $\begin{array}{l}\text { Intracellular membrane-bounded } \\
\text { organelle }\end{array}$ & 0.14 & 13 & $\begin{array}{l}\text { [Abhd10, Atp10d, Cblb, Flt4, Hmgn5, Masp1, Nnt, Nr1d1, Prox1, Serpina1c, Slc7a2, Sox15, } \\
\text { Steap4] }\end{array}$ \\
\hline
\end{tabular}

Note. The term $P$ value and the term $P$ value corrected with Bonferroni step-down $<0.05$ were considered. \%AGs and NGs correspond to the percent of the associated genes and the number of the involved genes respectively. The GO cellular component is the ontology source.

Table 3. Twenty-Three Biological Processes Which Are Related to a Number of Queried DEGs.

\begin{tabular}{|c|c|c|c|}
\hline GO Term & $\%$ AGs & NGs & Associated Genes Found \\
\hline Defense response & 0.68 & 9 & [Defb8, F3, II22ra1, Itgb6, Masp1, Nr1d1, Saa3, Slc7a2, Tspan6] \\
\hline Regulation of biological process & 0.17 & 16 & $\begin{array}{l}\text { [Cblb, Cd300lb, Cntfr, F3, Flt4, Hmgn5, Il22ra1, Itgb6, Masp1, Nr1d1, Prox1, Saa3, } \\
\text { Serpina1c, Slc7a2, Sox15, Tspan6] }\end{array}$ \\
\hline Response to stress & 0.37 & 12 & $\begin{array}{l}\text { [Defb8, F3, Flt4, Il22ra1, Itgb6, Masp1, Mmrn1, Nr1d1, Saa3, Slc7a2, Sox15, } \\
\text { Tspan6] }\end{array}$ \\
\hline Response to external stimulus & 0.44 & 9 & [Cd300lb, Defb8, Il22ra1, Nr1d1, Prox1, Saa3, Slc7a2, Sox15, Tspan6] \\
\hline Response to chemical & 0.22 & 8 & [Cd300lb, Cntfr, F3, Flt4, II22ra1, Nr1d1, Saa3, Serpina1c] \\
\hline Positive regulation of biological process & 0.21 & 11 & [Cblb, Cd300lb, Cntfr, F3, Flt4, Hmgn5, Masp1, Nr1d1, Prox1, Sox15, Tspan6] \\
\hline Negative regulation of biological process & 0.19 & 9 & [Cblb, Cntfr, Flt4, Hmgn5, Nr1d1, Prox1, Serpina1c, Sox15, Tspan6] \\
\hline Regulation of immune system process & 0.53 & 6 & [Cblb, Cd300lb, Masp1, Nr1d1, Slc7a2, Tspan6] \\
\hline Regulation of response to stimulus & 0.23 & 8 & [Cblb, F3, Flt4, Masp1, Nr1d1, Slc7a2, Sox15, Tspan6] \\
\hline Regulation of cellular process & 0.17 & 15 & $\begin{array}{l}\text { [Cblb, Cd300lb, Cntfr, F3, Flt4, Hmgn5, Il22ra1, Itgb6, Nr1d1, Prox1, Saa3, } \\
\text { Serpina1c, Slc7a2, Sox15, Tspan6] }\end{array}$ \\
\hline Signal transduction & 0.19 & 9 & [Cblb, Cntfr, F3, Flt4, Il22ra1, Itgb6, Nr1d1, Saa3, Tspan6] \\
\hline Response to organic substance & 0.29 & 8 & [Cd300lb, Cntfr, F3, Flt4, Il22ra1, Nr1d1, Saa3, Serpina1c] \\
\hline Regulation of cell proliferation & 0.52 & 8 & [Cblb, Cntfr, F3, Flt4, Hmgn5, Nr1d1, Prox1, Sox15] \\
\hline Positive regulation of cellular process & 0.19 & 9 & [Cd300lb, Cntfr, F3, Flt4, Hmgn5, Nr1d1, Prox1, Sox15, Tspan6] \\
\hline Negative regulation of cellular process & 0.21 & 9 & [Cblb, Cntfr, Flt4, Hmgn5, Nr1d1, Prox1, Serpina1c, Sox15, Tspan6] \\
\hline Positive regulation of response to stimulus & 0.37 & 7 & [Cblb, F3, Flt4, Masp1, Nr1d1, Sox15, Tspan6] \\
\hline Cellular response to chemical stimulus & 0.27 & 7 & [Cd300lb, Cntfr, F3, Flt4, Il22ra1, Nr1d1, Saa3] \\
\hline Organonitrogen compound metabolic process & 0.17 & 10 & [Cblb, F3, Flt4, Hmgn5, Masp1, Nnt, Nr1d1, Prox1, Saa3, Serpina1c] \\
\hline
\end{tabular}




\begin{tabular}{|c|c|c|c|}
\hline Positive regulation of cell proliferation & 0.71 & 6 & [Cntfr, F3, Flt4, Hmgn5, Prox1, Sox15] \\
\hline Cell surface receptor signaling pathway & 0.31 & 7 & [Cblb, Cntfr, F3, Flt4, II22ra1, Itgb6, Tspan6] \\
\hline $\begin{array}{l}\text { Positive regulation of macromolecule metabolic } \\
\text { process }\end{array}$ & 0.24 & 7 & [Cblb, F3, Flt4, Hmgn5, Nr1d1, Prox1, Sox15] \\
\hline $\begin{array}{l}\text { Positive regulation of nitrogen compound metabolic } \\
\text { process }\end{array}$ & 0.21 & 6 & [Cblb, F3, Flt4, Nr1d1, Prox1, Sox15] \\
\hline Cellular response to organic substance & 0.33 & 7 & [Cd300lb, Cntfr, F3, Flt4, Il22ra1, Nr1d1, Saa3] \\
\hline Immune System & 0.43 & 7 & [Cblb, Cd300lb, Cntfr, Il22ra1, Masp1, Serpina1c, Serpinb6d] \\
\hline
\end{tabular}

Note. The term $P$ value and the term $P$ value corrected with Bonferroni step-down $<0.05$ were considered. \%AGs and NGs correspond to the percent of the associated genes and the number of the involved genes respectively. The REACTOME Pathway and the GO biological process were the ontology source.

Table 4. Two Molecular Function Terms Related to 13 Queried DEGs.

\begin{tabular}{lccl}
\hline GO Term & $\%$ AGs & NGs & Associated Genes Found \\
\hline Receptor activity & 0.53 & 6 & [Cntfr, F3, Flt4, Il22ra1, Itgb6, Nr1d1] \\
Protein binding & 0.16 & 13 & [Cblb, Cd300lb, Cntfr, F3, Flt4, Il22ra1, Itgb6, Masp1, Nr1d1, Prox1, Saa3, Serpina1c, Sox15] \\
\hline
\end{tabular}

Note. The term $P$ value and the term $P$ value corrected with Bonferroni step-down $<0.05$ were considered. \%AGs and NGs correspond to the percent of the associated genes and the number of involved genes respectively. GO molecular function is the ontology source.

Table 5. Twenty-Six DEDs That Are Involved in the Biological Terms and the Frequency of the Related Biological Terms for the Individual DEGs Are Shown

\begin{tabular}{|c|c|c|}
\hline Gene Symbol & $\begin{array}{l}\text { Number of Involved } \\
\text { Biological Terms }\end{array}$ & $\begin{array}{c}\% \text { Frequency of Involved } \\
\text { Biological Terms }\end{array}$ \\
\hline Flt4 & 33 & 77 \\
\hline F3 & 33 & 77 \\
\hline Tspan6 & 29 & 67 \\
\hline Cntfr & 28 & 65 \\
\hline Nr1d1 & 27 & 73 \\
\hline Cblb & 23 & 53 \\
\hline $\operatorname{ltg} 66$ & 23 & 53 \\
\hline II22ra1 & 20 & 47 \\
\hline Sox 15 & 19 & 44 \\
\hline Cd300lb & 18 & 42 \\
\hline Steap4 & 17 & 40 \\
\hline Serpina 1c & 17 & 40 \\
\hline Prox 1 & 16 & 37 \\
\hline Masp1 & 16 & 37 \\
\hline Saa3 & 15 & 35 \\
\hline Slc13a3 & 15 & 35 \\
\hline Slc15a2 & 15 & 35 \\
\hline Slc7a2 & 15 & 335 \\
\hline Hmgn5 & 14 & 33 \\
\hline Atp 10d & 9 & 21 \\
\hline Nnt & 8 & 19 \\
\hline Defb8 & 3 & 07 \\
\hline Tmem $254 b$ & 3 & 07 \\
\hline Abhd10 & 3 & 07 \\
\hline Mmrn 1 & 2 & 05 \\
\hline Serpinb6d & 1 & 03 \\
\hline
\end{tabular}

regulated similarly. Also, it is shown that there is possible binding action between Tspan6 and Itgb6. Investigations into the role of exosomes in the promotion of important processes in intercellular communication and different types of diseases indicated that tetraspanin family proteins (Tspans) and integrins (Itga and Itgb) are key elements for process that exosomes select the recipient cells and bind to the surface of the cells. ${ }^{20}$ Itgb6 is linked to Flt4 by four types of actions including reciprocal activation, binding, reaction, and catalysis. The importance of dysregulation of integrins in different kinds of diseases is reported by researchers. Integrins are members of proteins that participate in the facility of signaling between the intracellular and extracellular environments. These proteins are cell-surface proteins and are formed from two hetrodimers. ${ }^{21-23}$

The central protein in Figure 3 is Flt4 which is connected to Itgb6, Cbl-b, and Il22ral. As it is shown in Table 2, Flt4 is up-regulated and it is the top participant protein in the identified biological terms (see Table 5). Flt4, as the receptor of vascular endothelial growth factor C (Vegfc) beside transcription factor Prox1 and Vegfc, plays an essential role in lymphatic system formation. Its dysregulation is tied to disorders such as Milroy, heart, and cardiovascular diseases. ${ }^{24-27}$ Prox 1 is the 13th element of Table 5 that is characterized by $37 \%$ of the "Frequency of the involved biological terms". As it is shown in Figure 3, Prox 1 activates Flt 4 and up-regulates its expression. Here, Cbl-b was the main gene, the direct effect of which on sensitivity against UVB irradiation was studied. The wide range of connections between Cbl-b and Flt4 (see Figure 3) reflects the key role of Flt4 in this study. However, there is a reciprocal activation connection between Cbl-b and Flt4 but Flt4 inhibits Cbl-b, the central hypothesis of this project. A binding action between Cbl-b and Itgb6 is highlighted in Figure 3, which links axis Itgb6-Tspan6 directly to Cbl-b. 
Since this project was administered with clear knowledge about the effect of Cbl-b on the promotion of radiation damages and the absence of a connection between F3Masplaxis and the core elements of the action map, it can be concluded that Flt4, Itgb6, and Tspan6 are critical genes in the protection of skin against UVB irradiation. On the other hand, Ftl4 plays a prominent role in the connection between Cbl-b and the other elements of the action map. Finally, Ftl4 is a suitable candidate which is the main responsible agent involved in the events related to the irradiated skin. It seems that the up-regulation of Flt4 is a protective process in response to the radiation.

\section{Conclusion}

It can be concluded that the protection process against UVB irradiation on skin implies gene expression changes in different types of genes. Our analysis revealed some biological terms which are related to the fundamental biological processes were affected by 26 genes. Screening methods showed Ftl4 was a central differential expressed gene which issued a significant regulatory property relative to the other critical genes. Compact regulatory action between Flt4 and neighbors corresponds to its prominent role in the protective response of the body against UVB irradiation.

\section{Ethical Considerations}

Not applicable.

\section{Conflict of Interests}

The authors declare no conflict of interest.

\section{Acknowledgments}

Shahid Beheshti University of Medical Sciences supports this research.

\section{References}

1. De Gruijl FR. Skin cancer and solar UV radiation. Eur J Cancer. 1999;35(14):2003-9. doi: 10.1016/S09598049(99)00283-X.

2. Bode AM, Dong Z. Mitogen-activated protein kinase activation in UV-induced signal transduction. Sci Signal. 2003;2003(167):re2. doi: 10.1126/stke.2003.167.re2.

3. Pasker-de Jong PC, Wielink G, van der Valk PG, van der Wilt GJ. Treatment with UV-B for psoriasis and nonmelanoma skin cancer: a systematic review of the literature. Arch Dermatol. 1999;135(7):834-40. doi: 10.1001/archderm.135.7.834.

4. Scherschun L, Kim JJ, Lim HW. Narrow-band ultraviolet $\mathrm{B}$ is a useful and well-tolerated treatment for vitiligo. $J$ Am Acad Dermatol. 2001;44(6):999-1003. doi: 10.1067/ mjd.2001.114752.

5. Ramsay DL, Lish KM, Yalowitz CB, Soter NA. Ultraviolet-B phototherapy for early-stage cutaneous T-cell lymphoma. Arch Dermatol. 1992;128(7):931-3.

6. Lee E, Koo J, Berger T. UVB phototherapy and skin cancer risk: a review of the literature. Int J Dermatol.
2005;44(5):355-60. doi: 10.1111/j.1365-4632.2004.02186.x.

7. Edgar R, Domrachev M, Lash AE. Gene Expression Omnibus: NCBI gene expression and hybridization array data repository. Nucleic Acids Res. 2002;30(1):207-10. doi: 10.1093/nar/30.1.207.

8. Babichev S, Škvor J. Technique of gene expression profiles extraction based on the complex use of clustering and classification methods. Diagnostics (Basel). 2020;10(8):584. doi: 10.3390/diagnostics10080584.

9. Zamanian-Azodi M, Rezaei-Tavirani M, Rezaei Tavirani M. Investigating the effects of ibuprofen on the gene expression profile in Hippocampus of mice model of Alzheimer's disease through bioinformatics analysis. Iran J Pharm Res. 2020;19(2):352-9. doi: 10.22037/ijpr.2019.15485.13125.

10. Amiri-Dashatan N, Rezaei-Tavirani M, Ahmadi N. A quantitative proteomic and bioinformatics analysis of proteins in metacyclogenesis of Leishmania tropica. Acta Trop. 2020;202:105227. doi: 10.1016/j. actatropica.2019.105227.

11. Rostami-Nejad M, Razzaghi Z, Esmaeili S, Rezaei-Tavirani S, Akbarzadeh Baghban A, Vafaee R. Immunological reactions by $\mathrm{T}$ cell and regulation of crucial genes in treated celiac disease patients. Gastroenterol Hepatol Bed Bench. 2020;13(2):155-60. doi: 10.22037/ghfbb.v13i2.1921.

12. Manikyam HK. Computational studies on Gene Ontology for Molecular functions, Cellular component and Biological process of SARS-CoV-2 targeted proteins, 04 May 2020, PREPRINT (Version 1) Available at Research Square: https://doi.org/10.21203/rs.3.rs-26263/v1.

13. Barghi N, Bambaeichi E, Rezaei-Tavirani M, Khaledi N. Aerobic exercises induce antioxidant pathways activation in rats. Int J Prev Med. 2020;11:144. doi: 10.4103/ijpvm. IJPVM_246_19.

14. Singh TP, Vieyra-Garcia PA, Wagner K, Penninger J, Wolf P. Cbl-b deficiency provides protection against UVB-induced skin damage by modulating inflammatory gene signature. Cell Death Dis. 2018;9(8):835. doi: 10.1038/s41419-0180858-5.

15. Rezaei Tavirani M, Rezaei Tavirani S, Tajik Rostami F. Biochemical pathway analysis of gastric atrophy. Gastroenterol Hepatol Bed Bench. 2018;11(2):118-24.

16. Karbalaei R, Piran M, Rezaei-Tavirani M, AsadzadehAghdaei H, Heidari MH. A systems biology analysis protein-protein interaction of NASH and IBD based on comprehensive gene information. Gastroenterol Hepatol Bed Bench. 2017;10(3):194-201.

17. Brand RM, Wipf P, Durham A, Epperly MW, Greenberger JS, Falo Jr LD. Targeting mitochondrial oxidative stress to mitigate UV-induced skin damage. Front Pharmacol. 2018;9:920. doi: 10.3389/fphar.2018.00920.

18. Balestrin LA, Bidone J, Bortolin RC, Moresco K, Moreira JC, Teixeira HF. Protective effect of a hydrogel containing Achyrocline satureioides extract-loaded nanoemulsion against UV-induced skin damage. J Photochem Photobiol B. 2016;163:269-76. doi: 10.1016/j.jphotobiol.2016.08.039.

19. Mohania D, Chandel S, Kumar P, Verma V, Digvijay K, Tripathi D, et al. Ultraviolet radiations: Skin defensedamage mechanism. In: Ahmad SI, ed. Ultraviolet Light in Human Health, Diseases and Environment. Cham, Switzerland: Springer; 2017. p. 71-87.

20. Dilsiz N. Role of exosomes and exosomal microRNAs in 
cancer. Future Sci OA. 2020;6(4):FSO465. doi: 10.2144/ fsoa-2019-0116.

21. Meecham A, Marshall JF. The ITGB6 gene: its role in experimental and clinical biology. Gene X. 2020;5:100023. doi: 10.1016/j.gene.2019.100023.

22. Dotan I, Allez M, Danese S, Keir M, Tole S, McBride J. The role of integrins in the pathogenesis of inflammatory bowel disease: Approved and investigational anti-integrin therapies. Med Res Rev. 2020;40(1):245-62. doi: 10.1002/ med.21601.

23. Zhang Y, Reif G, Wallace DP. Extracellular matrix, integrins, and focal adhesion signaling in polycystic kidney disease. Cell Signal. 2020;72:109646. doi: 10.1016/j. cellsig.2020.109646.

24. Gauvrit S, Villasenor A, Strilic B, Kitchen P, Collins MM, Marín-Juez R, et al. HHEX is a transcriptional regulator of the VEGFC/FLT4/PROX1 signaling axis during vascular development. Nat Commun. 2018;9(1):2704. doi: 10.1038/ s41467-018-05039-1.

25. Sui Y, Lu Y, Lin M, Ni X, Chen X, Li H, et al. A Milroy Disease Family Caused by FLT4 Gene Mutation of c.2774 T\&gt;A with Phenotypes Heterogeneity, 05 October 2020, PREPRINT (Version 1). Available at Research Square: https://doi.org/10.21203/rs.3.rs-44896/v1.

26. Reuter MS, Chaturvedi RR, Liston E, Manshaei R, Aul RB, Bowdin S, et al. The cardiac genome clinic: implementing genome sequencing in pediatric heart disease. Genet Med. 2020;22(6):1015-1024. doi: 10.1038/s41436-020-0757-x.

27. Bhattacharjee S, Lee $\mathrm{Y}$, Zhu B, Wu H, Chen Y, Chen $\mathrm{H}$. Epsins in vascular development, function and disease. Cell Mol Life Sci. 2020. doi: 10.1007/s00018-020-03642-4. 